\title{
Correction to: Kidney health for everyone everywhere: from prevention to detection and equitable access to care
}

\author{
Philip Kam-Tao $\mathrm{Li}^{1}$. Guillermo Garcia-Garcia ${ }^{2}$ S Siu-Fai Lui ${ }^{3}$. Sharon Andreoli ${ }^{4}$. Winston Wing-Shing Fung ${ }^{1}$. \\ Anne Hradsky ${ }^{5}$. Latha Kumaraswami ${ }^{6}$. Vassilios Liakopoulos ${ }^{7} \cdot$ Ziyoda Rakhimova $^{5} \cdot$ Gamal Saadi $^{8} \cdot$ Luisa Strani $^{5}$. \\ Ifeoma Ulasi ${ }^{9} \cdot$ Kamyar Kalantar-Zadeh $^{10}$. for the World Kidney Day Steering Committee
}

Published online: 16 July 2020

(c) Copyright by the World Kidney Day 2020 Steering Committee. The article was originally published in Kidney International. The permission of republication herein was granted by the authors, the journal and its publisher 2020

\section{Correction to: Journal of Nephrology (2020) 33:201-210 https://doi.org/10.1007/s40620-020-00728-x}

The copyright of the article has been incorrectly published.

The copyright line for the article should read:

CCopyright by the World Kidney Day 2020 Steering Committee. The article was originally published in Kidney International. The permission of republication herein was granted by the authors, the journal and its publisher.

Publisher's Note Springer Nature remains neutral with regard to jurisdictional claims in published maps and institutional affiliations.

The original article can be found online at https://doi.org/10.1007/ s40620-020-00728-x.

Philip Kam-Tao Li

philipli@cuhk.edu.hk

$\triangle$ Kamyar Kalantar-Zadeh

kkz@uci.edu

1 Department of Medicine and Therapeutics, Carol and Richard Yu PD Research Centre, Prince of Wales Hospital, Chinese University of Hong Kong, 30-32 Ngan Shing Street, Shatin, New Territories, Hong Kong, China

2 Nephrology Service, Hospital Civil de Guadalajara Fray Antonio Alcalde, University of Guadalajara Health Sciences Center, Guadalajara, Jal., Mexico

3 Division of Health System, Policy and Management, Jockey Club School of Public Health and Primary Care, The Chinese University of Hong Kong, Hong Kong, China

4 James Whitcomb Riley Hospital for Children, Indiana University School of Medicine, Indianapolis, USA
5 World Kidney Day Office, Avenue des Arts 1-2, 6th floor, 1210 Brussels, Belgium

6 Tanker Foundation, Chennai, India

7 Division of Nephrology and Hypertension, 1st Department of Internal Medicine, AHEPA Hospital, Aristotle University of Thessaloniki, Thessaloniki, Greece

8 Nephrology Unit, Department of Internal Medicine, Faculty of Medicine, Cairo University, Giza, Egypt

9 Renal Unit, Department of Medicine, College of Medicine, University of Nigeria, Ituku-Ozalla, Enugu, Nigeria

10 Division of Nephrology and Hypertension and Kidney Transplantation, University of California Irvine School of Medicine, Orange, CA, USA 